\title{
A copula based model for multivariate non normal longitudinal data: analysis of a dose titration safety study on a new antidepressant
}

\author{
Philippe Lambert ${ }^{1, *}$ and François Vandenhende ${ }^{1,2}$ \\ ${ }^{1}$ Université Catholique de Louvain, Institut de Statistique, Voie du Roman Pays 20, \\ B-1348 Louvain-la-Neuve, Belgium. \\ ${ }^{2}$ Statistics \& Information Sciences, Eli Lilly \& Company, \\ B-1348 Mont-Saint-Guibert, Belgium.
}

September 18, 2001

SUMMARY

A new model for multivariate non normal longitudinal data is proposed. In a first step, each longitudinal series of data corresponding to a given response is modelled separately using a copula to relate the marginal distributions of the response at each time of observation.

In a second step, at each observation time, the conditional (on the past) distributions of each response are related using another copula describing the relationship between the corresponding variables. Note that there is no need to consider the same family of distributions for these response variables.

The technique is illustrated in a dose titration safety study on a new antidepressant. The hemodynamic effect on diastolic blood pressure, systolic blood pressure and heart rate is studied. These three responses are measured repeatedly over time on 10 healthy volunteers during the dose escalation. The available covariates are sex and the concentration of drug in the plasma at time of measurement.

${ }^{*}$ Correspondence to: Philippe Lambert, Université Catholique de Louvain, Institut de Statistique, Voie du Roman Pays 20, B-1348 Louvain-la-Neuve (Belgium). E-mail: lambert@stat.ucl.ac.be Phone: +32-10-47.28.01 Fax: +32-10-47.30.32 


\section{INTRODUCTION}

Modelling multivariate longitudinal data is an important challenge in biostatistics. In the literature, most of the time, the considered models assume that the original data or some transformation of them are multivariate normal with a variancecovariance matrix structured [1] to describe serial dependence, heterogeneity and dependence between response variables (see Section 4.1). Unfortunately, normality is certainly not a rule in practice. When the responses are not normal or/and when their marginal distributions are not in the same family, alternatives to the multivariate normal must be found. In this paper, we shall focus on longitudinal data of mixed types.

Very few tools exist in the literature to model such data. Zeger and Liang ${ }^{2}$ consider, at each time point $t$, a model for the mean of each outcome conditional on its history and on the values of the other outcomes at time $t$. The conditional variance of each outcome is assumed proportional to a fixed function of the conditional mean. An extension of the generalized estimating equations (GEE, [3]) method is used to estimate parameters.

Jørgensen, Lundbye-Christensen, Song and $\mathrm{Sun}^{4}$ propose state-space models based on the class of Tweedie exponential dispersion models. They can deal with a few discrete distributions, namely the Poisson and the compound Poisson, and several continuous distributions among which the normal, the gamma and the inverse Gauss. Their vectors of observations observed repeatedly at equally spaced times are assumed conditionally independent given a univariate latent process.

More tools can be found in the literature to deal with data of mixed types when the vectors of observations are only observed once on independent or clustered units.

A joint model for mixed continuous and categorical data was proposed by Olkin and Tate. ${ }^{5}$ It was extended to deal with missing values in [6]. Conditionally on the categorical responses combination (with a marginal multinomial distribution), they 
assume that the continuous variables are multivariate normal. The EM algorithm [7] is used to obtain the maximum likelihood estimators (MLEs) in the presence of missing values. Liu and Rubin ${ }^{8}$ generalized the approach by considering a multivariate $t$ distribution instead of the more constraining multivariate normal and by allowing different covariance matrices across cells.

Many models for mixed type data can be found in the literature devoted to the analysis of toxicity studies. These usually involve clustered responses of mixed types [9]. One example is in reproduction toxicolgy. The examination of the fetuses of an exposed pregnant mice provides data on the presence of fetus malformation, fetal death and fetal weight. These response variables of mixed types can then be related to dam size and exposure in a single model.

Fitzmaurice and Laird ${ }^{10}$ proposed to model such data using marginal regression models for each of the responses while treating the intra-cluster correlation as a nuisance. GEEs are derived to obtain consistent parameter estimates while accounting for intra-cluster correlation. The parameters from the marginal regression models are robust to dependence misspecification.

Another possibility would be to express the joint distribution of two (say) responses as the product of one marginal distribution and of the conditional distribution of the other response given the first one. The choice of the conditioning response is of course arbitrary. See [11] for a model in which a binary response is modelled conditionally on a continuous normal response and [12] for a discussion of the above factorization strategy.

In a similar problem, Catalano and $\mathrm{Ryan}^{13}$ proposed to model the discrete outcome using a latent variable. They considered a bivariate normal distribution for the latent variable and the continuous response.

The same type of approach with a single conditioning latent variable was used by Sammel, Ryan and Legler ${ }^{14}$ to relate mixed type outcomes modelled using gen- 
eralized linear models. It was used to analyse the effect of an anticonvulsant on the development of birth defects.

Regan and Catalano ${ }^{15}$ showed how to build a joint model for clustered binary and continuous outcomes with an application to developmental toxicology. They use an extension of the correlated probit model [16] to obtain a joint distribution for the binary and the continuous responses. A joint multivariate normal distribution is considered for the corresponding latent variables and each outcome is analysed with a marginal dose-response model. The covariance matrix takes into account the correlation between outcomes and the correlation due to clustering. This is an important improvement of [13] and [10] as model estimates of the correlation between responses and the evolution of these correlations with dose are available. A marginal approach was preferred to conditional models with the arguments that the biological mechanism relating the chemical exposition to developmental problems is not well understood, and that conditional models are difficult to use to quantify risk or to study the marginal effect of dose on an isolated response.

In this work, we propose to show how copulas can be used to model multivariate non-normal longitudinal data. By definition, a $n$-dimensional copula $C\left(u_{1}, \ldots, u_{n}\right)$ is a cumulative distribution function with uniform marginals $[17,18,19]$. If $F^{(j)}\left(y_{j}\right)$ is the distribution of a continuous univariate random variable $Y_{j}(j=1, \ldots, n)$, then $C\left(F^{(1)}\left(y_{1}\right), \ldots, F^{(n)}\left(y_{n}\right)\right)$ is a multivariate distribution for $\boldsymbol{Y}=\left(Y_{1}, \ldots, Y_{n}\right)^{T}$ with marginal distributions $F^{(j)}(j=1, \ldots, n)$. Conversely, it can be proved that for any multivariate distribution $F\left(y_{1}, \ldots, y_{n}\right)$ with continuous marginal distributions $F^{(j)}(j=1, \ldots, n)$, there exists a copula $C\left(u_{1}, \ldots, u_{n}\right)$ such that

$$
F\left(y_{1}, \ldots, y_{n}\right)=C\left(F^{(1)}\left(y_{1}\right), \ldots, F^{(n)}\left(y_{n}\right)\right)
$$

Originally, these results were mainly used to simulate multivariate random variables with given marginal distributions. More recently, they have been considered to model dependent data in subjects like survival analysis [20], genetics [21], risk theory 
[22] and insurance [23].

The plan of the paper is as follows. In Section 2, we present the study that motivated this research. Section 3 is devoted to notation. A copula [17] model for univariate longitudinal (possibly) non normal data is proposed in Section 4 where the normal case with a structured covariance matrix is described in detail. An extension to non normal data is then derived. A joint model for all the series which accounts for the dependence between all the responses is described in Section 5. We conclude the paper by the analysis of the motivating study in Section 6 and by a discussion in Section 7.

\section{THE MOTIVATING STUDY}

The goal of this dose titration study is to learn about the hemodynamic effect of a new antidepressant. The considered compound, an inhibitor of norepinephrine and serotonin uptake, is indicated in the treatment of depression.

A subject blind, placebo controlled, randomised study was performed on 12 volunteers (6 males and 6 females) to assess the safety, pharmacokinetics and pharmacodynamics of this drug during a dose escalation phase. One male and one female were on the placebo arm, the other ten volunteers being on the treatment arm. For them, the dose administered was progressively incremented: $20 \mathrm{mg}$ twice daily (b.i.d.) on days $1-2,40 \mathrm{mg}$ b.i.d. on days $3-8,60 \mathrm{mg}$ b.i.d. on days $9-14,80 \mathrm{mg}$ b.i.d. on days $15-19$ and $80 \mathrm{mg}$ on the morning of day 20 . An abrupt treatment discontinuation was performed on day 20 and monitored up to day 22 . The administration of the drug occured in the morning at 8 a.m. and in the evening at 8 p.m.

Our analysis will focus on safety data and more precisely on the assessment of the effect of the drug on heart rate (HR, in beats/min), diastolic (DBP) and systolic blood pressures (SBP) (in $\mathrm{mmHg}$ ) for the ten subjects in the treatment arm. 
These measurements were taken before the first dose on day 1 and 4 hours after the morning dose on days $6-8,12-14,18-22$. Thus, twelve repeated measurements were recorded per subject for each of the three response variables. In addition, the drug concentration (in $\mathrm{ng} / \mathrm{ml}$ ) was measured in plasma at the same times. An additional covariate is sex. Plots of the three responses against time can be found in Figures 1, 2 and 3 for the ten subjects.

In the subsequent analysis, we shall use the concentration of the dose found in plasma instead of the actual dose to evaluate the effect of the drug on the three responses. Indeed, plasma concentrations are more reliable measures of exposure than the administered dose during a dose-escalation phase and when steady state conditions are not yet reached. Moreover, a direct association between hemodynamic response and systemic exposure is possible because the two components are measured in the same cardiovascular system.

\section{NOTATION}

Let $\left\{t_{j}: j=1, \ldots, J\right\}$ be the $J$ (possibly unequally spaced) measurement times and $i(i=1, \ldots, I=10)$ index the $I$ subjects in the study.

For any subject $i$ at time $t_{j}$, suppose that we measure $R$ responses $\left(y_{i j}^{(r)}\right)_{r=1, \ldots, R}$. In most of the text, the subject subscript $i$ and the response superscript $r$ will be dropped to simplify the equations. Unless explicitly stated, equations will always refer to any of the $R$ responses observed on any given subject at the indicated time point. This will apply in most of Section 4 . The considered response will usually be indicated in Section 5 to stress that parameters are not necessarily shared by the $R$ marginal models for the responses. 


\section{A STATISTICAL MODEL FOR UNIVARIATE LONGITUDINAL DATA}

Let us consider each of the $R$ responses separately. The multivariate aspect of the problem will be addressed in a later section. To fix ideas, consider the $J$ measures of response $r$ on subject $i$. As already mentioned, $i$ and $r$ will usually be dropped to simplify notation.

\subsection{The normal model}

The procedure that we propose starts from the well known multivariate normal modelling strategy used in repeated measurements theory (see for example [24]). If normality is believed to be a reasonable hypothesis, a possible model for $\boldsymbol{Y}=$ $\left(Y_{1}, \ldots, Y_{J}\right)^{T}$ could be

$$
\boldsymbol{Y} \sim M V N_{J}(\boldsymbol{\mu}, \boldsymbol{\Sigma})
$$

where $\boldsymbol{\mu}=\left(\mu_{j}\right)_{j=1, \ldots, J}$ is the $J$-vector of response means and $\boldsymbol{\Sigma}=\left(\sigma_{j k}\right)_{1 \leq j, k \leq J}$ is the variance-covariance matrix. Note that the subscript (here $J$ ) used when denoting distributions and densities just indicates the dimension of the corresponding random variable.

The mean can vary with covariates in a way to be defined. The response variance can also be modelled as a function of covariates. Let us denote by $\beta$ the parameters used to describe the covariates effect on the mean and on the variance. Thus, at time $t_{j}$, we have

$$
\mu_{j}=\mu\left(\boldsymbol{x}_{j} ; \boldsymbol{\beta}\right) ; \sigma_{j j}=\sigma_{j}^{2}=\sigma^{2}\left(\boldsymbol{x}_{j} ; \boldsymbol{\beta}\right)
$$

where $\boldsymbol{x}_{j}$ are the measured covariates at that time for the considered subject.

Two potential sources of dependence in repeated measures are considered:

Individual heterogeneity: measurements of a response realized on the same individual tend to be more alike than across individuals. 
Serial dependence: measurements of a response realized on the same individual at close time points tend to be more closely related than measurements made at more distant times.

The correlation matrix $\boldsymbol{R}=\left(\rho_{j k}\right)_{1 \leq j, k \leq J}$ that we assume identical for all subjects, is parametrized to account for these two types of dependence in longitudinal data. A possible choice for $\boldsymbol{R}=\boldsymbol{R}\left(\rho_{h}, \rho_{s}\right)$ is

$$
\rho_{j k}=\rho_{j k}\left(\rho_{h}, \rho_{s}\right)=\rho_{h}+\left(1-\rho_{h}\right) \rho_{s}^{\left|t_{k}-t_{j}\right|} \text { for } k \neq l
$$

where $\left|\rho_{h}\right| \leq 1$ and $\left|\rho_{s}\right| \leq 1$ with the additional constraint that $R\left(\rho_{h}, \rho_{s}\right)$ must be positive definite. We have $\rho_{j k} \rightarrow 1$ as $\left|t_{k}-t_{j}\right| \rightarrow 0$ and $\rho_{j k} \rightarrow \rho_{h}$ as $\left|t_{k}-t_{j}\right| \rightarrow \infty$. Thus, as the time interval between 2 measurements increases, a residual intra-unit correlation $\rho_{h}$ due to individual heterogeneity remains. An extra correlation term $\left(1-\rho_{h}\right) \rho_{s}^{\Delta t}$ arises when the time interval $\Delta t$ is small.

Note that the variance-covariance matrix $\boldsymbol{\Sigma}$ is a consequence of the chosen models for the response variance and for the correlation matrix as

$$
\begin{aligned}
\sigma_{j j} & =\sigma_{j}^{2}=\sigma^{2}\left(\boldsymbol{x}_{j} ; \boldsymbol{\beta}\right) \\
\sigma_{j k} & =\rho_{j k}\left(\rho_{h}, \rho_{s}\right) \sigma\left(\boldsymbol{x}_{j} ; \boldsymbol{\beta}\right) \sigma\left(\boldsymbol{x}_{k} ; \boldsymbol{\beta}\right)
\end{aligned}
$$

Estimators of the regression parameters $\boldsymbol{\beta}$ and of the correlation parameters $\left(\rho_{h}, \rho_{s}\right)$ can be obtained by maximizing the likelihood

$$
L(\boldsymbol{\theta})=\prod_{i} L_{i}(\boldsymbol{\theta})=\prod_{i} \phi_{J}\left(y_{i 1}-\mu_{i 1}, \ldots, y_{i J}-\mu_{i J} \mid \Sigma_{i}\right)
$$

where

- $\boldsymbol{\theta}=\left(\boldsymbol{\beta}^{T}, \rho_{h}, \rho_{s}\right)^{T}$

- $L_{i}(\boldsymbol{\theta})$ is the likelihood contribution due to subject $i$,

- $\mu_{i j}=\mu\left(\boldsymbol{x}_{i j} ; \boldsymbol{\beta}\right)$ and $\Sigma_{i}=\Sigma_{i}(\boldsymbol{\theta})$, 
- $\phi_{J}(\cdot \mid \Sigma)$ denotes the density of a normally distributed $J$-variate with mean vector 0 and variance-covariance matrix $\Sigma$.

A multivariate non normal distribution where the dependence structure can be specified as easily and as clearly as in the normal case is clearly lacking in the statistical literature. This is precisely the type of model that we propose below.

\subsection{Rewriting of the multivariate normal model}

Let us rewrite the likelihood (2) in such a way that the normality assumption made for the univariate marginal distribution $F_{1}\left(y_{i j} \mid \boldsymbol{\beta}\right)$ (with mean $\mu_{i j}$ and variance $\left.\sigma_{i j}^{2}\right)$ ) of each response at time $t_{j}$ clearly comes out. Let us drop index $i$ to simplify notation. If $f_{1}\left(y_{j} \mid \boldsymbol{\beta}\right)$ denotes the corresponding univariate density and $f_{J}\left(y_{1}, \ldots, y_{J} \mid \boldsymbol{\theta}\right)$ the joint density of the $J$ repeated measurements on a given subject, then the likelihood contribution due to that subject is

$$
\begin{aligned}
L(\boldsymbol{\theta}) & =f_{J}\left(y_{1}, \ldots, y_{J} \mid \boldsymbol{\theta}\right) \\
& =\phi_{J}\left(y_{1}-\mu_{1}, \ldots, y_{J}-\mu_{J} \mid \Sigma(\boldsymbol{\theta})\right) \\
& =\frac{1}{\prod_{j=1}^{J} \sigma_{j}} \phi_{J}\left(\frac{y_{1}-\mu_{1}}{\sigma_{1}}, \ldots, \frac{y_{J}-\mu_{J}}{\sigma_{J}} \mid R\left(\rho_{h}, \rho_{s}\right)\right) \\
& =K^{\prime}\left(F_{1}\left(y_{1} \mid \boldsymbol{\beta}\right), \ldots, F_{1}\left(y_{J} \mid \boldsymbol{\beta}\right) \mid R\left(\rho_{h}, \rho_{s}\right)\right) \prod_{j=1}^{J} f_{1}\left(y_{j} \mid \boldsymbol{\beta}\right)
\end{aligned}
$$

where

$$
K^{\prime}\left(u_{1}, \ldots, u_{J} \mid R\right)=\frac{\partial^{J}}{\partial u_{1} \ldots \partial u_{J}} K\left(u_{1}, \ldots, u_{J} \mid R\right)
$$

is the derivative of the normal copula (see [18], p. 140)

$$
K\left(u_{1}, \ldots, u_{J} \mid R\right)=\Phi_{J}\left(\Phi_{1}^{-1}\left(u_{1}\right), \ldots, \Phi_{1}^{-1}\left(u_{n}\right) \mid R\right)
$$

yielding

$$
K^{\prime}\left(u_{1}, \ldots, u_{J} \mid R\right)=\frac{\phi_{J}\left(\Phi_{1}^{-1}\left(u_{1}\right), \ldots, \Phi_{1}^{-1}\left(u_{n}\right) \mid R\right)}{\prod_{j=1}^{J} \phi_{1}\left(\Phi_{1}^{-1}\left(u_{j}\right)\right)}
$$

with 
- $\Phi_{1}(\cdot)$ and $\phi_{1}(\cdot)$ denoting respectively the distribution and the density of a standardized univariate normal variate.

- $\Phi_{J}(\cdot \mid \Sigma)$ and $\phi_{J}(\cdot \mid \Sigma)$ denoting the distribution and the density of a normal $J$-variate with mean 0 and variance-covariance matrix $\Sigma$.

Under independence, i.e. under the assumption that $\rho_{h}=\rho_{s}=0$, the first factor in Equation (3) (corresponding to the derivative of the copula) is 1 and the likelihood reduces to the product of the (marginal) densities at the $J$ time points.

\subsection{A non normal longitudinal model}

We propose to relax the normality hypothesis made for the marginal distribution of the response at each time $t_{j}$ and to consider instead any continuous distribution that might be found appropriate.

If $F_{1}\left(y_{j} \mid \boldsymbol{\beta}\right)$ denotes that distribution and $f_{1}\left(y_{j} \mid \boldsymbol{\beta}\right)$ the corresponding density, then Equation (3) determines a multivariate continuous distribution (for the $J$ repeated measurements of the considered response for the chosen subject) with marginal continuous distribution $F_{1}\left(y_{j} \mid \boldsymbol{\beta}\right)$ at time $t_{j}$ and dependence parameters $\rho_{h}$ and $\rho_{s}$, zero values yielding independence. Note that, unlike in the multivariate normal model, $\rho_{j k}\left(\rho_{h}, \rho_{s}\right)$ from Equation (1) is not the correlation between $Y_{j}$ and $Y_{k}$ anymore, although it still measures the strength of the dependence between these two variables. Indeed, it can be shown (see e.g. [18], p. 54) that the Kendall's tau $\tau_{j k}$ corresponding to $\rho_{j k}$ is

$$
\tau_{j k}=\frac{2}{\pi} \arcsin \left(\rho_{j k}\right)
$$

As plotted in Figure 4, the dependence between $Y_{j}$ and $Y_{k}$ is strictly increasing with $\rho_{j k}$

Note that Kendall's tau is not sensitive to monotonic transformations of the data. As we shall consider different marginal distributions for the response in the 
modelling of our data (see Section 6.1.1), the estimation that we shall obtain for $\rho_{h}$ and $\rho_{s}$ should not be too much influenced by the choice made for the marginals.

Other copulas than the normal could be used if judged appropriate. Popular parametric specifications can be found in [18, Chap. 5].

\section{A MODEL FOR MULTIVARIATE LONGITUDINAL DATA}

The models in Section 4 treat the $R$ responses separately as if they were independent. In this section, we propose to relate these responses using a copula to connect their conditional (on the past) distributions at each time point.

We start by rewriting the likelihood for each of the $R$ responses as the product of conditional distributions. For the $r$ th response and a given subject, we obtain

$$
\begin{aligned}
L^{(r)}\left(\boldsymbol{\theta}^{(r)}\right) & =f_{J}^{(r)}\left(y_{1}^{(r)}, \ldots, y_{J}^{(r)} \mid \boldsymbol{\theta}^{(r)}\right) \\
& =f_{1}^{(r)}\left(y_{1}^{(r)} \mid \boldsymbol{\theta}^{(r)}\right) f_{1}^{(r)}\left(y_{2}^{(r)} \mid \mathcal{F}_{2^{-}}^{(r)}, \boldsymbol{\theta}^{(r)}\right) \ldots f_{1}^{(r)}\left(y_{J}^{(r)} \mid \mathcal{F}_{J^{-}}^{(r)}, \boldsymbol{\theta}^{(r)}\right)
\end{aligned}
$$

with

$$
f_{1}^{(r)}\left(y_{j}^{(r)} \mid \mathcal{F}_{j^{-}}^{(r)}, \boldsymbol{\theta}^{(r)}\right)=\frac{f_{j}^{(r)}\left(y_{1}^{(r)}, \ldots, y_{j}^{(r)} \mid \boldsymbol{\theta}^{(r)}\right)}{f_{j-1}^{(r)}\left(y_{1}^{(r)}, \ldots, y_{j-1}^{(r)} \mid \boldsymbol{\theta}^{(r)}\right)}
$$

where $\mathcal{F}_{j^{-}}^{(r)}$ denotes the history of the $r$ th response (i.e. the vector of past responses with all pertinent relationships among them) of the considered subject up to (but not including) time $t_{j}$. The expressions for the joint densities in the numerator and denominator of Equation (5) are given by Equation (3) with marginal distributions $F_{j}^{(r)}$ and parameters $\boldsymbol{\theta}^{(r)}$ specific to the $r$ th response model.

At each time point $t_{j}$ for a given subject, we observe $\left(y_{j}^{(1)}, \ldots, y_{j}^{(R)}\right)$ for the $R$ responses. The likelihood associated to the $R$ series for this subject is

$$
h_{R}\left(y_{1}^{(1)}, \ldots, y_{1}^{(R)}\right) \prod_{j=2}^{J} h_{R}\left(y_{j}^{(1)}, \ldots, y_{j}^{(R)} \mid \mathcal{F}_{j^{-}}\right)
$$

where $h_{R}(\cdot)$ is the $R$-variate density associated to the $R$ responses and $\mathcal{F}_{j^{-}}$denotes the history of the $R$ responses (for the subject) up to (but not including) time $t_{j}$. 
Let us introduce an assumption on the dependence between the $R$ responses: we suppose that the rth response at time $t_{j}$ is, conditionally on its history and conditionally on the other responses at time $t_{j}$, independent of the history of these other responses. In other words, we assume that we can explain the effect of past responses on the $r$ th response by the history of the $r$ th response and by the current values of the other responses.

Under that hypothesis, we have as conditional (on the considered subject's response history) distribution for the $R$ response variables at time $t_{j}$

$$
\begin{gathered}
H_{R}\left(Y_{j}^{(1)} \leq y_{j}^{(1)}, \ldots, Y_{j}^{(R)} \leq y_{j}^{(R)} \mid \mathcal{F}_{j^{-}}\right)= \\
C_{\boldsymbol{\alpha}}\left(F_{1}^{(1)}\left(y_{j}^{(1)} \mid \mathcal{F}_{j^{-}}^{(1)}, \boldsymbol{\theta}^{(1)}\right), \ldots, F_{1}^{(R)}\left(y_{j}^{(R)} \mid \mathcal{F}_{j^{-}}^{(R)}, \boldsymbol{\theta}^{(R)}\right)\right)
\end{gathered}
$$

where $C \boldsymbol{\alpha}\left(u_{1}, \ldots, u_{R}\right)$ is the copula (with dependence parameter vector $\boldsymbol{\alpha}$ ) relating the conditional (on their respective history) distributions of the $R$ responses at time $t_{j}$

The corresponding conditional density (obtained by taking partial derivatives of the expression in Equation (7)) can be used in Equation (6) to obtain the contribution to the likelihood for the chosen subject. This likelihood is a function of the vector of parameters $\boldsymbol{\theta}$ where

$$
\boldsymbol{\theta}^{T}=\left(\boldsymbol{\theta}^{(1)^{T}}, \ldots, \boldsymbol{\theta}^{(R)^{T}}, \boldsymbol{\alpha}^{T}\right)
$$

\section{STUDY ANALYSIS}

\subsection{Univariate responses}

Let us first analyse the evolution of diastolic BP, systolic BP and heart rate separately. 


\subsubsection{Modelling strategy}

We have considered different parametric marginal distributions $F_{1}\left(y_{j} \mid \boldsymbol{\beta}\right)$ for each response. The considered possibilities were the gamma, the Weibull, the inverse Gauss, the normal, the log-normal, the Student and the log-Student.

The chosen location parameter $\mu_{j}$ and the dispersion parameter $\sigma_{j}$ of these marginal models were allowed to change with sex and the drug concentration in the plasma. Consider for example the Student distribution. The most general model that we have considered is

$$
\begin{aligned}
\mu_{j} & =\lambda_{0}+\lambda_{1} \operatorname{conc}_{j} \\
\log \left(\sigma_{j}\right) & =\delta_{0}+\Delta \delta_{C} \mathrm{I}_{\left\{\operatorname{conc}_{j} \neq 0\right\}}+\delta_{1} \operatorname{conc}_{j}
\end{aligned}
$$

with possibly different parameters for males and females. The tail parameter (degree of freedom) $v$ was assumed fixed. Hence, referring to the above notation and taking male as the reference category,

$$
\boldsymbol{\beta}=\left(\lambda_{0}^{M}, \Delta \lambda_{0}^{F}, \lambda_{1}^{M}, \Delta \lambda_{1}^{F}, \delta_{0}^{M}, \Delta \delta_{0}^{F}, \Delta \delta_{C}, \delta_{1}^{M}, \Delta \delta_{1}^{F}, v\right)^{T}
$$

where, for example,

$$
\lambda_{0 F}=\lambda_{0 M}+\Delta \lambda_{0 F}
$$

is used to obtain the intercept in the location parameter equation for females.

The maximum likelihood estimates (MLEs) of $\boldsymbol{\theta}=\left(\boldsymbol{\beta}^{T}, \rho_{h}, \rho_{s}\right)^{T}$ were calculated by a numerical maximization of the likelihood with subject contribution given by Equation (3). The function nlm in the free software R [25] was used to do this, yielding the required parameter estimates and standard errors. Model selection was realized using the Akaike criterion [26]. Note that there is no allowance for uncertainty in the selection of the marginal distributions for the three responses. We refer to [27] for a description of the problem and a possible Bayesian answer. 


\subsubsection{Results}

The selected longitudinal model for each of the three response series is reported in Table 1. The number in square brackets gives the change in deviance $(=-2 \log (L))$ when the corresponding parameter

- is fixed (usually) at zero when it is estimated in the table,

- is estimated when it is (usually) fixed at zero in the table.

This is given together with standard errors (in brackets) as we cannot discard non symmetric likelihood profiles for some of the involved parameters ; using standard errors in such situations can be misleading. As the AIC was used to do model selection, any parameter causing a decrease in the deviance of at least 2 is maintained in the model.

Note that time does not appear explicitly associated with a regression parameter in the table. Indeed, time is only used to describe serial dependence between the repeated measurements as explained in Section 4.1 and $\rho_{s}$ was only found necessary to model heart rate profiles. In this dose escalation study, drug concentration tends to increase with time. For this reason, the effect of time appears indirectly in the model as it is associated with the variation of the drug concentration in plasma.

Let us detail the selected model for each of the three response variables.

Diastolic BP The selected marginal distribution is Normal. This marginal distribution describes how the diastolic blood pressure of the subjects involved in the study is distributed conditionally on the amount of drug found in their plasma. The considered location and dispersion parameters in Equation (8) are the mean and the standard deviation.

No sex effect was detected on the marginal mean. However, the mean DPB increases significantly with the drug concentration.

The dispersion is larger when the drug concentration is not zero, i.e. after 
administration of the drug, and this difference increases with the drug concentration.

There is some evidence of intra-subject association $\left(\hat{\rho}_{h}=0.68 \Rightarrow \hat{\tau}_{h}=0.48\right)$ but not of serial dependence. In others words, the DBP measurements tend to be more homogeneous when they are realized on the same individual, but the association remains identical whatever the time interval (considered in the study) separating responses.

The evolution of the fitted (marginal) quantiles for a range of drug concentrations can be found in Figure 5.

Systolic BP A log-Student distribution with 7.14 for the degree of freedom is selected. The marginal distribution of the SBP is thus right skewed with a heavier tail than a log-normal, indicating that very large values for the SBP cannot be discarded. Note that a log-normal distribution is selected instead if the very large systolic BP measured for one of the subject on day 7 (see e.g. Figure 2) is removed. However, we have decided to maintain that observation in the dataset as it was really measured. Considering a Student distribution instead of the traditional normal for the log of the systolic BP remains a cautious choice at it allows to make a (parametric) robust inference on the mean response (see [28] for a similar argument in another context): outliers mainly affect the degree of freedom.

The considered location and dispersion parameters in Equation (8) are the mean of the $\log$ SBP and the standard deviation of the log SBP.

There is a small increase in the marginal mean (of the log SBP) with the concentration too, but no significant sex effect.

No significant sex or concentration effect was found on dispersion.

As for the DBP, there is evidence of intra-subject association $\left(\hat{\rho}_{h}=0.56 \Rightarrow \hat{\tau}_{h}=\right.$ 
0.38) in the SBP, but not of serial association.

The evolution of the fitted (marginal) quantiles for a range of drug concentrations can be found in Figure 6.

Heart rate Heart rate was treated as if it were continuous. The selected distribution is the gamma. The considered parametrisation for the gamma density is

$$
f(y)=\frac{1}{(\phi \mu)^{\frac{1}{\phi}} \Gamma\left(\frac{1}{\phi}\right)} x^{\frac{1}{\phi}-1} \mathrm{e}^{-\frac{1}{\phi} \frac{x}{\mu}}
$$

such that

$$
\mathrm{E}(Y)=\mu \text { and } \mathrm{V}(Y)=\phi \mu^{2}
$$

The considered location and dispersion parameters in Equation (8) are the mean $\mu$ and the parameter $\phi$ of the gamma conditional distribution for the heart rate.

The marginal mean heart rate is significantly smaller for men than for women. It is not significantly related to the drug concentration.

However, the scale parameter (which is estimated to be $0.026\left(=\mathrm{e}^{\hat{\delta}_{0 M}}\right)$ when no treatment is given $)$ tends to decrease $\left(\hat{\delta}_{1 M}=0 ; \hat{\delta}_{1 F}=-0.0049\right)$ for females (but not for males) as the drug concentration increases.

There is evidence of an intra-subject association $\left(\hat{\rho}_{h}=0.67 \Rightarrow \hat{\tau}_{h}=0.46\right)$. Serial dependence was also significant $\left(\hat{\rho}_{s}=0.30\right)$. The Kendall's tau measuring the association between two heart rate measurements on the same subject is thus at least equal to $\hat{\tau}_{h}$. The necessity to keep the serial association parameter in the model contrasts with the results found for the DBP and the SBP.

The evolution of the fitted (marginal) quantiles for a range of drug concentrations can be found in Figure 7. 


\subsection{Multivariate responses}

The use of copulas allows to separate the modelling of the three responses and of their dependence. We have already modelled the three series separately in Section 6.1 .

We shall use these results with Equations (5) and (7) to obtain the conditional distributions required to get the likelihood contribution of subject $i$ as given by Equation (6).

The choice of the copula $C_{\boldsymbol{\alpha}}\left(u_{1}, u_{2}, u_{3}\right)$ is arbitrary and should, as much as possible, reflect the dependence structure between the three responses. Here, again, we decided to restrict our choice to the normal copula as the dependence structure can easily be specified through the variance-covariance matrix. The dependence between any two of the three responses measured by a parameter $\rho$ (with $|\rho| \leq 1$ ) is again related to Kendall's tau by Equation (4).

Of course, other three-variate copulas could be used without difficulty if judged necessary. We refer the interested reader to [18] and [19] for a review of useful copulas and of their properties.

Thus, we have

$$
C_{\boldsymbol{\alpha}}\left(u_{1}, u_{2}, u_{3}\right)=\Phi_{3}\left(\Phi_{1}^{-1}\left(u_{1}\right), \Phi_{1}^{-1}\left(u_{2}\right), \Phi_{1}^{-1}\left(u_{3}\right) \mid \boldsymbol{R}(\boldsymbol{\alpha})\right)
$$

where

- $\boldsymbol{\alpha}^{T}=\left(\alpha^{D S}, \alpha^{D H}, \alpha^{S H}\right)$

- $\Phi_{3}(\cdot \mid \boldsymbol{R}(\boldsymbol{\alpha}))$ denotes the 3-variate normal distribution with mean 0 and covariance matrix

$$
\boldsymbol{R}(\boldsymbol{\alpha})=\left(\begin{array}{ccc}
1 & \alpha^{D S} & \alpha^{D H} \\
\alpha^{D S} & 1 & \alpha^{S H} \\
\alpha^{D H} & \alpha^{S H} & 1
\end{array}\right)
$$


These (between responses) dependence parameters were allowed to change with sex and presence of drug in the plasma. If $\alpha_{i j}$ denotes such a parameter for unit $i$ at time $t_{j}$, the most general (or $f u l l$ ) model considered for $\alpha_{i j}$ is

$$
\log \left(\frac{1+\alpha_{i j}}{1-\alpha_{i j}}\right)=\kappa_{0}+\Delta \kappa_{C} \mathrm{I}_{\left\{\operatorname{conc}_{i j} \neq 0\right\}}
$$

with separate parameters for males and females. Note that $\log \left(\frac{1+\alpha_{i j}}{1-\alpha_{i j}}\right)$ is a strictly increasing function of $\alpha_{i j}$, with the same sign as $\alpha_{i j}$. It tends to $+\infty(-\infty)$ when $\alpha_{i j}$ tends to $+1(-1)$. A significantly non zero value for $\Delta \kappa_{C}$ in Equation (9) indicates a significant drug effect. When it is positive (negative), it indicates that the dependence between the two considered responses significantly increases (decreases) when there is drug in the plasma. Independence occurs when both $\kappa_{0}$ and $\Delta \kappa_{C}$ are zero.

The MLEs (and standard errors) of the between response dependence parameters associated to the full model (including four parameters for each of the three possible pairs of responses) are reported in Table 2. The model was simplified by setting some of these parameters equal to zero or by constraining others to be the same for males and females. Model selection was again performed using the AIC. The MLEs associated to the selected model can be found in Table 3. A number in square brackets gives the change in deviance $(=-2 \log (L))$ when the corresponding parameter

- is fixed (usually) at zero when estimated in the table,

- is estimated when fixed in the table.

The fitted Kendall's tau corresponding to the selected multivariate model can be found in Table 4. They were obtained using Equations (4), (9) and the estimates in Table 3.

We can conclude the following:

Heart rate-Systolic BP association There is no significant association between 
these two variables.

Heart rate-Diastolic BP association There is a significant positive association between these two variables with a fitted Kendall's tau equal to

- 0.53 before treatment,

- 0.07 when there is drug in the plasma. Although it is small, it is significantly different from zero.

There is no significant effect of sex on the association between HR and DBP. These results are illustrated in Figures 8 and 9. They give the univariate and bivariate marginal distributions of $\mathrm{HR}$ and SBP of a male before treatment, in Figure 8, and after treatment (with, say, $35 \mathrm{ng} / \mathrm{mml}$ of drug in the plasma), in Figure 9. By comparing the two figures, we clearly see that the DBP is more dispersed after treatment while the heart rate distribution remains nearly unchanged. The positive association between the two responses is visible in both figures, although very moderate on the second. This moderate association (corresponding to a Kendall's tau close to zero) cannot be imputed to the increase in the DBP dispersion as Kendall's tau is insensitive to monotone and hence to scale transformations of the considered variables.

Systolic BP-Diastolic BP association There is a significant positive association between these two variables with a fitted Kendall's tau equal to

- 0 and 0.42 for females before and after drug administration respectively.

- 0.22 for males. No significant treatment effect on the association parameter was detected. 


\section{DISCUSSION}

In this study, the three responses were treated as if they were multivariate continuous although the heart rate is discrete. This choice was not due to a limitation of the proposed tool. Indeed, copulas can be used whatever the type and the marginal distribution of the involved variables. But when non continuous variables are involved, one must consider finite differences instead of derivatives of the copula to write down the likelihood. Therefore, the above model can also be used with multivariate longitudinal data of mixed types.

The normal copula was used throughout the paper for simplicity. Indeed, by specifying two correlation matrices (which should be named more generally dependence matrices), we have defined entirely the dependence arising due to the repeated character of our measurements with the first copula and the dependence between the different types of measurements at each time point with the second copula. But nothing prevents us from using other copulas than the normal one. We refer the interested reader to [18] and [19] for alternative multivariate copulas. For example, Vandenhende and Lambert (Discussion paper $25^{1}$, UCL, Belgium) use the multivariate Frank copula to model longitudinal ordinal data.

Except when considering bivariate Archimedian copulas [29], descriptive and graphical tools are clearly lacking to understand the dependence structure in the data and to make a preselection of appropriate copulas. This is the subject of ongoing work that we plan to publish later. For the moment, we select our copula(s) in simple parametric families (indexed by one or two parameters) by taking the one giving the largest log-likelihood.

The estimation of the parameters (from the second copula) describing the dependence between the different variables was made conditionally on the fitted univariate marginal distributions. This uses the argument that continuous margins in a copula

\footnotetext{
${ }^{1}$ http://www.stat.ucl.ac.be/ISpublication/ISdp.html
} 
model do not depend on the choice of the dependence structure, suggesting that the modelling and the parameter estimations for the margins and the copula can be made separately. One can show (see [18], p. 299) that this suggestion can be correct when working with normal copulas.

The drug concentration in the plasma was used as covariate instead of the administered dose for reasons exposed in Section 2. Of course, as soon as the way to administer the medication takes a definitive form and the dosage strength is known, a model relating the responses to the administered dose will become desirable. A pharmacokinetic model relating the drug concentration in the plasma to the administered dose could be developed to become the first building block of the final model, the above model being used as the second block. Note that sex will certainly play a key role in the pharmacokinetics model as the systemic concentrations were usually larger and more variable in women. This fact is illustrated in Figure 10. Sex did not play a key role as covariate in the models of Sections 6.1 and 6.2 where a drug concentration effect was already present.

\section{REFERENCES}

1. Wolfinger, R. 'Heterogeneous variance-covariance structures for repeated measures', Journal of Agricultural, Biological, and Environmental Statistics, 1, 205-230 (1986).

2. Zeger, S. and Liang, K. 'Feedback models for discrete and continuous time series', Statistica Sinica, 1, 51-64 (1991).

3. Liang, K. and Zeger, S. 'Longitudinal data analysis using generalized linear models', Biometrika, 73, 12-22 (1986).

4. Jørgensen, B., Lundbye-Christensen, S., Song, P. X.-K. and Sun, L. 'State-space models for multivariate longitudinal data of mixed types', Canadian Journal of Statistics, 24, 385-402 (1996).

5. Olkin, I. and Tate, R. 'Multivariate correlation models with mixed discrete and continuous variables', Annals of Mathematical Statistics, 32, 448-465 (1961). 
6. Little, R. and Schluchter, M. 'Maximum likelihood estimation for mixed continuous and categorical data with missing values', Biometrika, 72, 497-512 (1985).

7. Dempster, A., Laird, N. and Rubin, D. 'Maximum likelihood from incomplete data via the EM algorithm', Journal of the Royal Statistical Society, Series B, 39, 1-38 (1977)

8. Liu, C. and Rubin, D. 'Ellipsoidally symmetric extensions of the general location model for mixed categorical and continuous data', Biometrika, 85, 673-688 (1998).

9. Ryan, L. 'Statistical issues in toxicology', Journal of the American Statistical Association, 95, 304-308 (2000).

10. Fitzmaurice, G. and Laird, N. 'Regression models for a bivariate discrete and continuous outcome with clustering', Journal of the American Statistical Association, 90, 845-852 (1995).

11. Cox, D. 'The analysis of multivariate binary data', Applied Statistics, 21, 113-120 (1972).

12. Cox, D. and Wermuth, N. 'Response models for mixed binary and quantitative variables', Biometrika, 79, 441-461 (1992).

13. Catalano, P. and Ryan, L. 'Bivariate latent variable models for clustered discrete and continuous outcomes', Journal of the American Statistical Association, 87, 651-658 (1992).

14. Sammel, M., Ryan, L. and Legler, J. 'Latent variable models for mixed discrete and continuous outcomes', Journal of the Royal Statistical Society, Series B, 59, 667-678 (1997).

15. Regan, M. and Catalano, P. 'Likelihood models for clustered binary and continuous outcomes: application to developmental toxicology', Biometrics, 55, 760-768 (1999).

16. Ochi, Y. and Prentice, R. 'Likelihood inference in a correlated probit regression model', Biometrika, 71, 531-543 (1984).

17. Sklar, A. 'Fonctions de répartitions à $n$ dimensions et leurs marges', Publ. Inst. Statist. Univ. Paris, 8, 229-231 (1959). 
18. Joe, H. Multivariate Models and Dependence Concepts, Chapman \& Hall, London, 1997.

19. Nelsen, R. An Introduction to Copulas, Springer-Verlag, New York, 1998.

20. Hougaard, P. Analysis of Multivariate Survival Data, Springer-Verlag, New-York, 2000.

21. Trégouet, D., Ducimetiere, P., Bocquet, V., Visvikis, S., Soubrier, F. and Tiret, L. 'A parametric copula model for analysis of familial binary data', American Journal of Human Genetics, 64, 886-893 (1999).

22. Clemen, R. and Reilly, T. 'Correlations and copulas for decision and risk analysis', Management science, 45, 208-224 (1999).

23. Frees, E., Carriere, J. and Valdez, E. 'Annuity valuation with dependent mortality', Journal of Risk and Insurance, 63, 229-261 (1996).

24. Potthoff, R. and Roy, S. 'A generalized multivariate analysis of variance model useful especially for growth curve problems', Biometrika, 51, 313-326 (1964).

25. Ihaka, R. and Gentleman, R. 'R: a language for data analysis and graphics', Journal of Computational Graphics and Statistics, 5, 299-314 (1996).

26. Akaike, H. Information theory and an extension of the maximum likelihood principle, in B. Petrov and F. Csáki (eds), 'Second International Symposium on Inference Theory, Budapest', pp. 267-281, (1973).

27. Draper, D. 'Assessment and propagation of model uncertainty', Journal of the Royal Statistical Society, Series B, 57, 45-97 (1995).

28. Lambert, P. and Lindsey, J. 'Analysing financial returns using regression models based on non-symmetric stable distributions', Applied Statistics, 48, 409-424 (1999).

29. Genest, C. and Rivest, L.-P. 'Statistical inference procedures for bivariate archimedian copulas', Journal of the American Statistical Association, 88, 1034-1043 (1993). 




Figure 1: Diastolic BP (in $\mathrm{mmHg}$ ) 4 hours after the dose administration. 


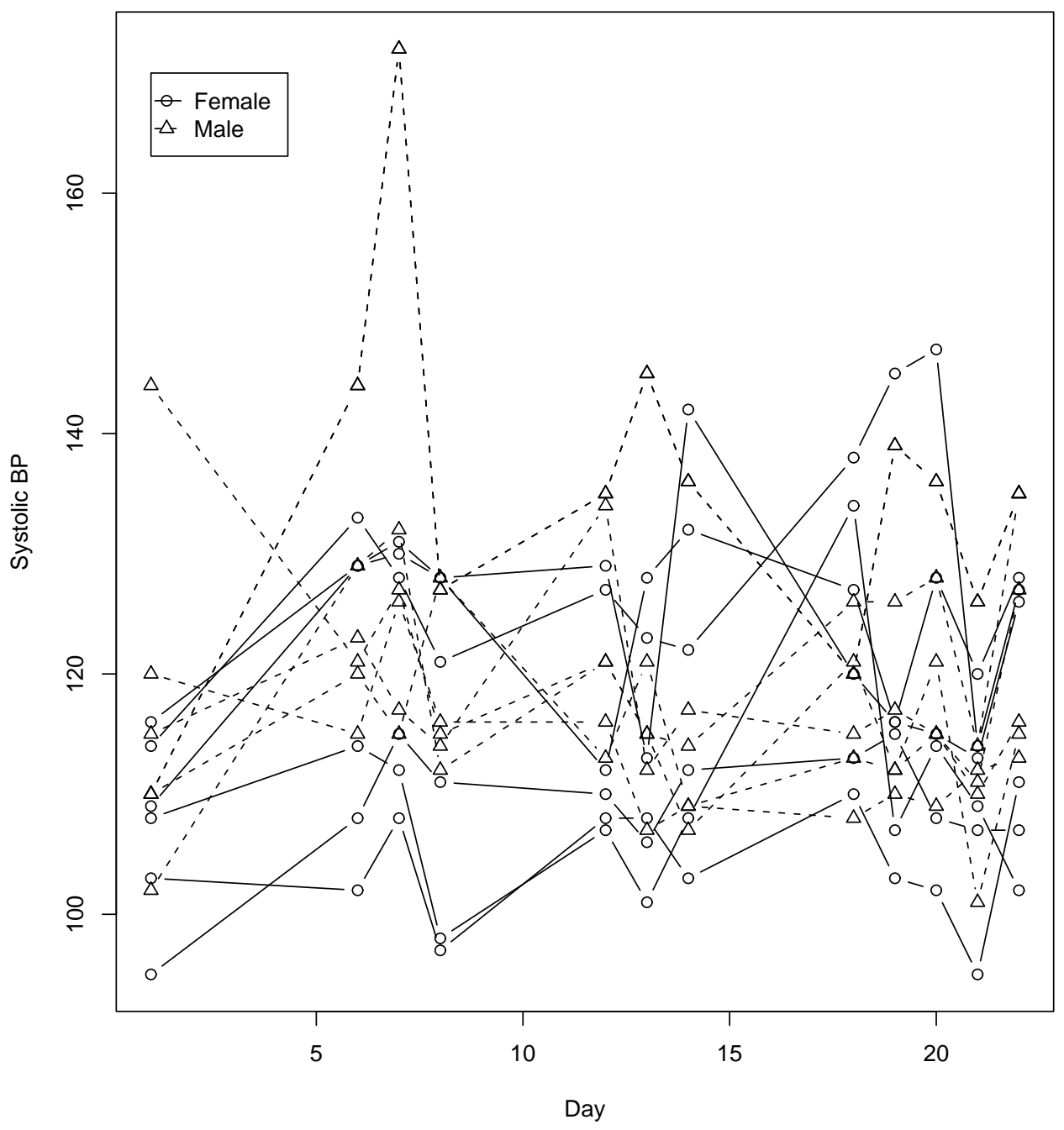

Figure 2: Systolic BP (in $\mathrm{mmHg}$ ) 4 hours after the dose administration. 


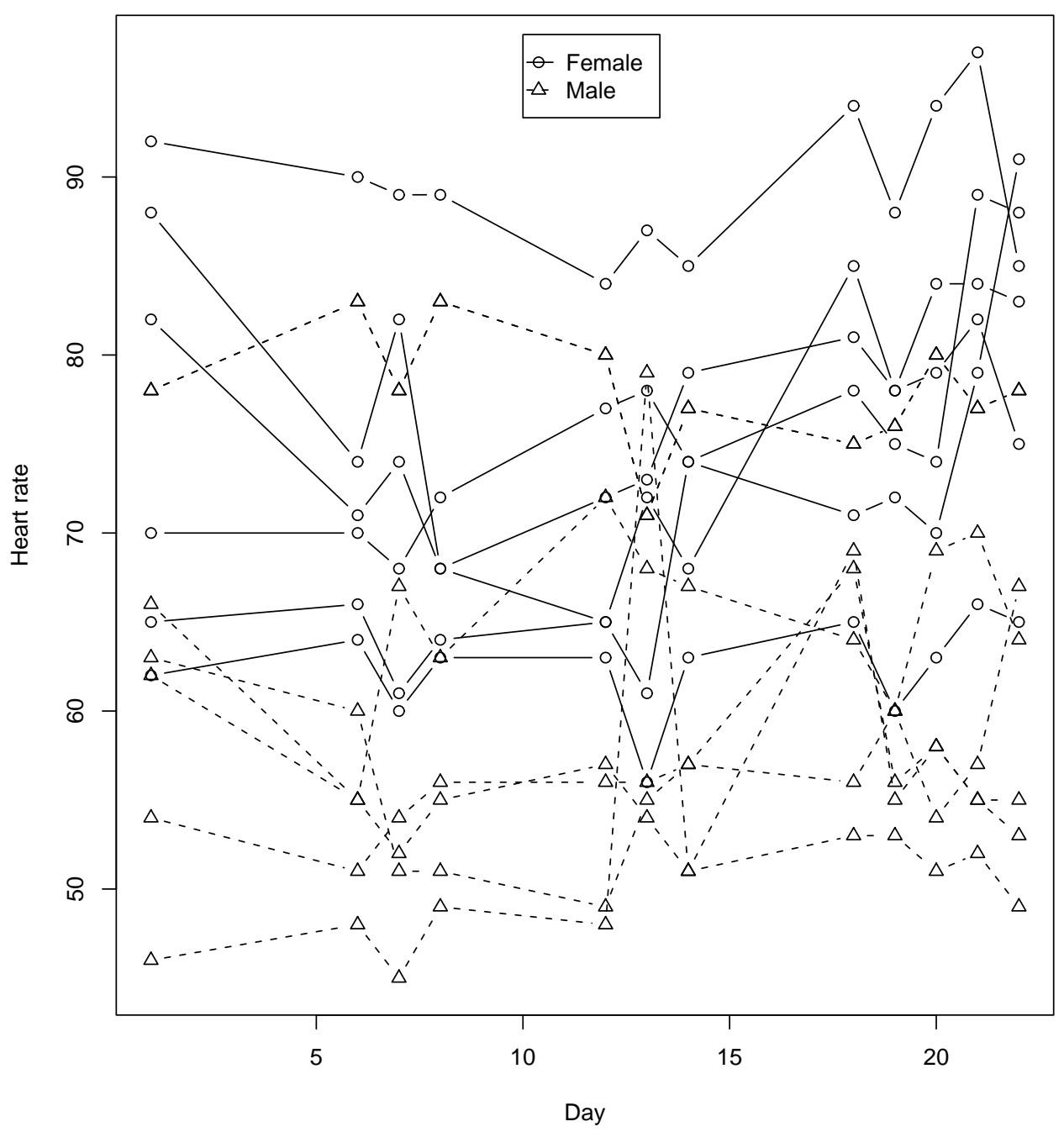

Figure 3: Heart rate (in beats/min) 4 hours after the dose administration. 


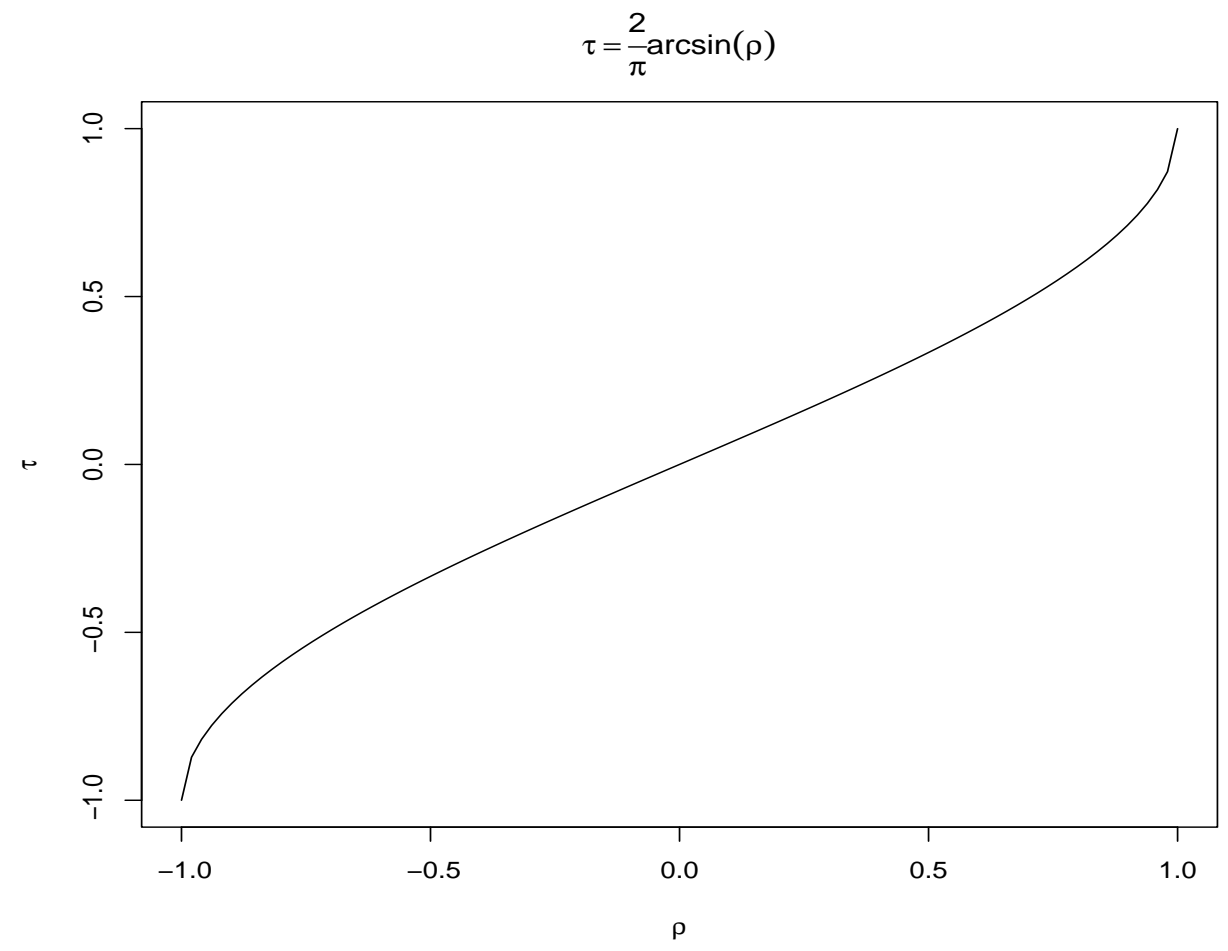

Figure 4: Relationship between the correlation coefficient and Kendall's tau in a bivariate normal model. 


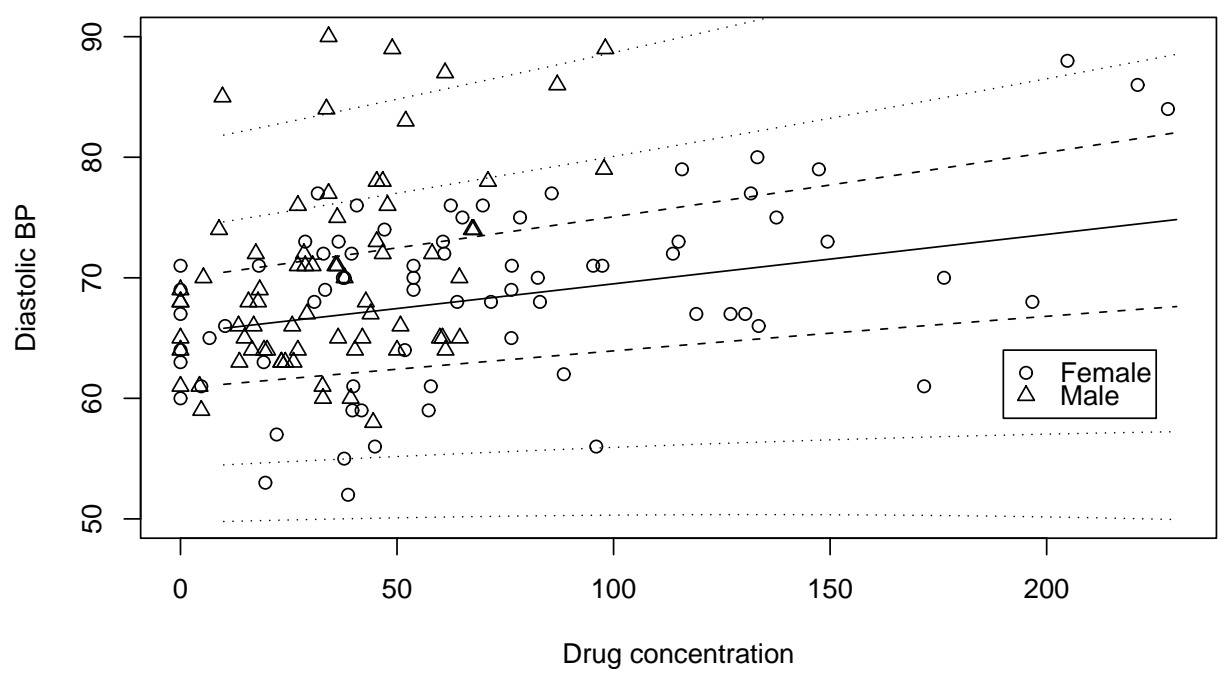

Figure 5: Effect of the drug concentration in the plasma (in $\mathrm{ng} / \mathrm{ml}$ ) on the quantiles $0.01,0.10,0.25,0.50,0.75,0.90$ and 0.99 of the marginal normal distribution of the (supine) diastolic blood pressure (in $\mathrm{mmHg}$ ). 


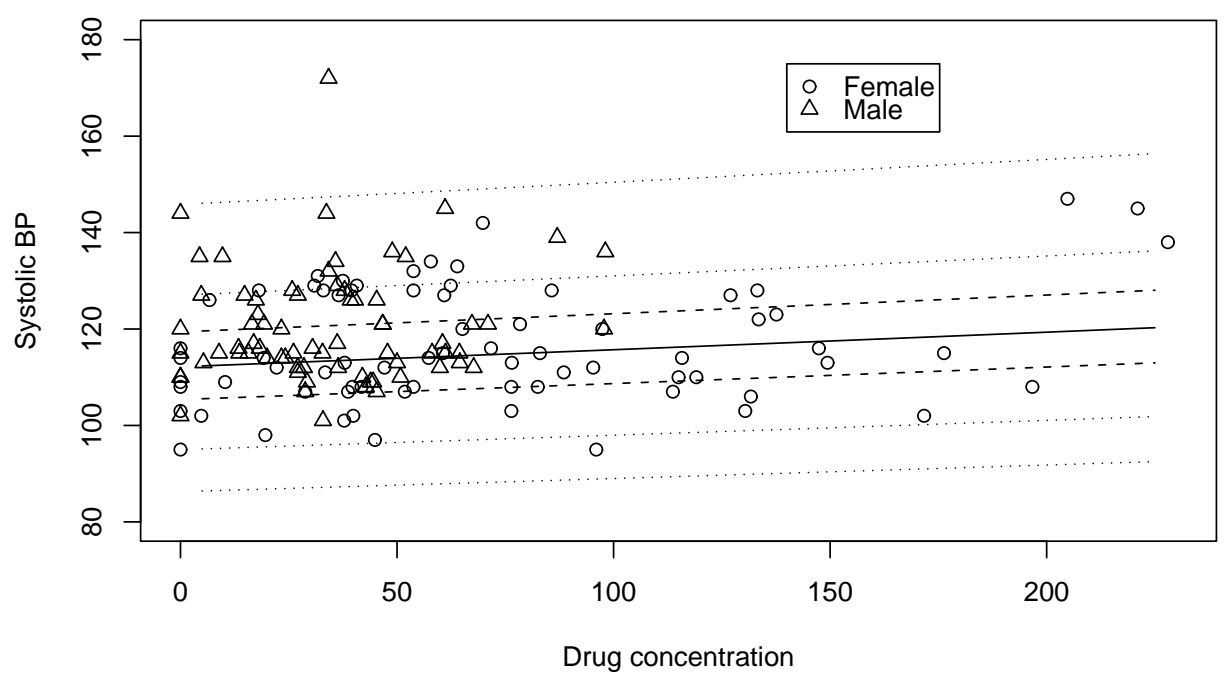

Figure 6: Effect of the drug concentration in the plasma (in $\mathrm{ng} / \mathrm{ml}$ ) on the quantiles $0.01,0.10,0.25,0.50,0.75,0.90$ and 0.99 of the marginal log-Student distribution of the (supine) systolic blood pressure (in $\mathrm{mmHg}$ ). 

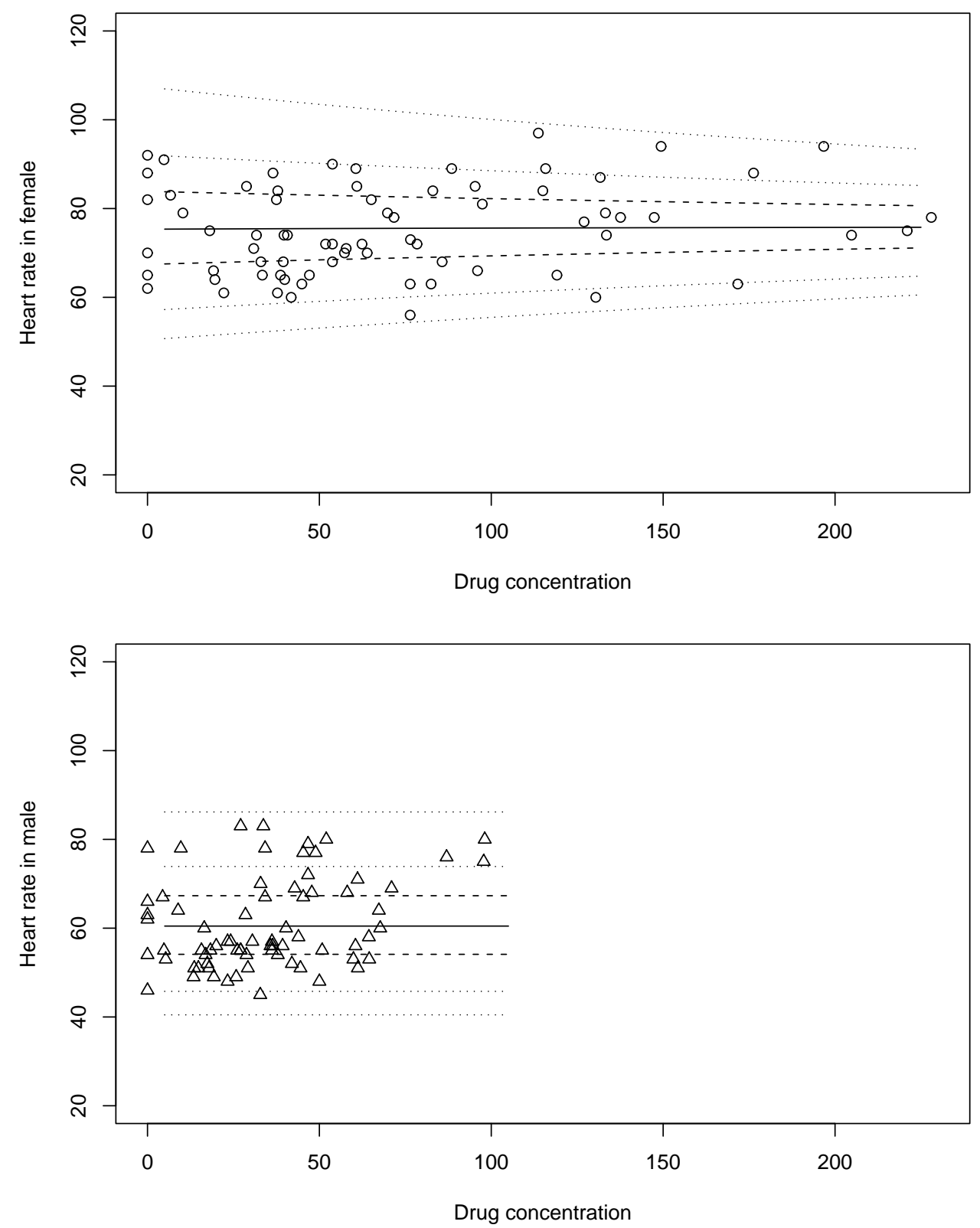

Figure 7: Effect of the drug concentration in the plasma (in $\mathrm{ng} / \mathrm{ml}$ ) on the quantiles $0.01,0.10,0.25,0.50,0.75,0.90$ and 0.99 of the marginal gamma distribution of the (supine) heart rate (in beats/min). 

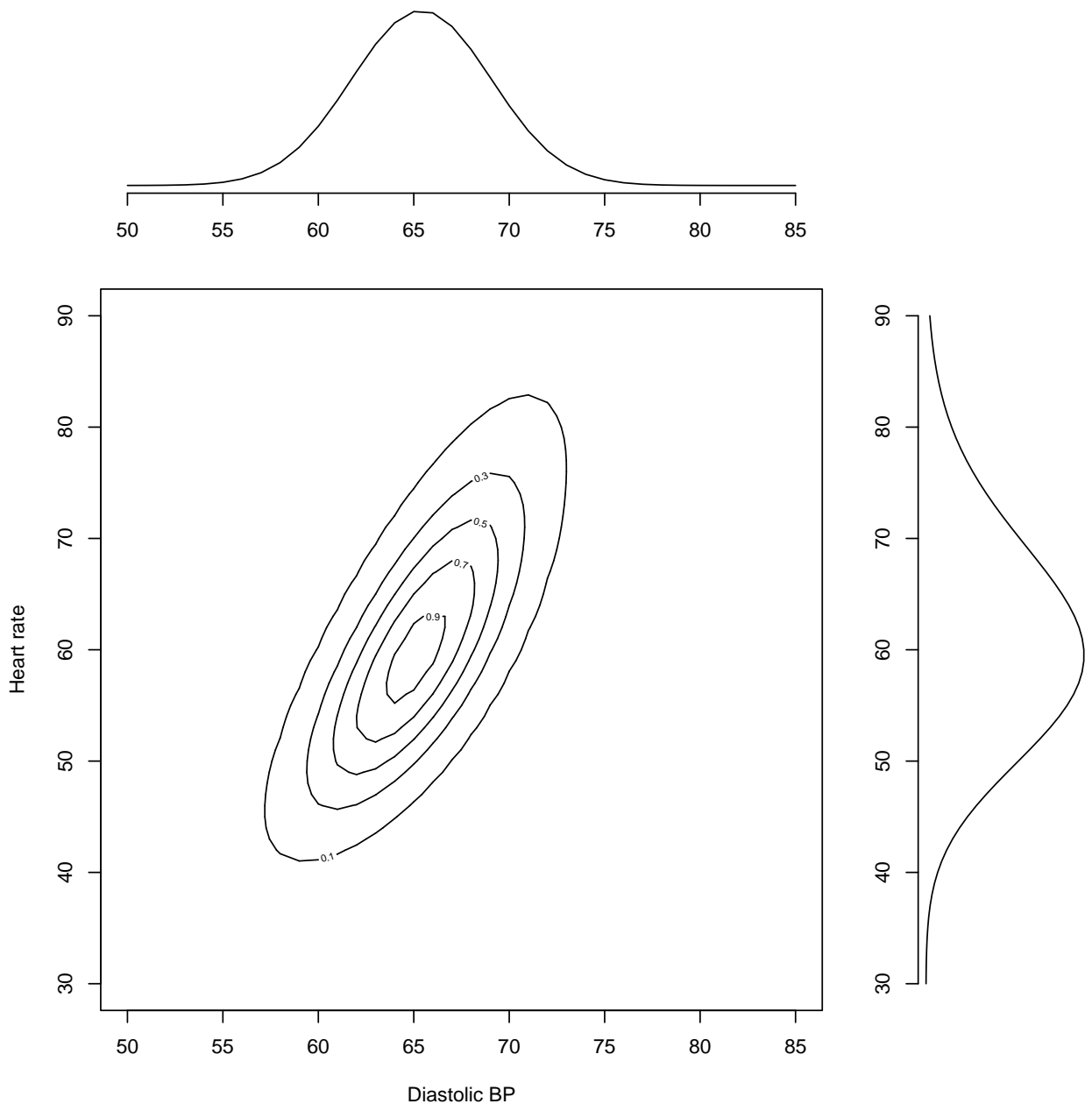

Figure 8: Fitted univariate and bivariate marginal distributions of the heart rate (in beats/min) and of the diastolic $\mathrm{BP}$ (in $\mathrm{mmHg}$ ) for a male without drug in the plasma. 

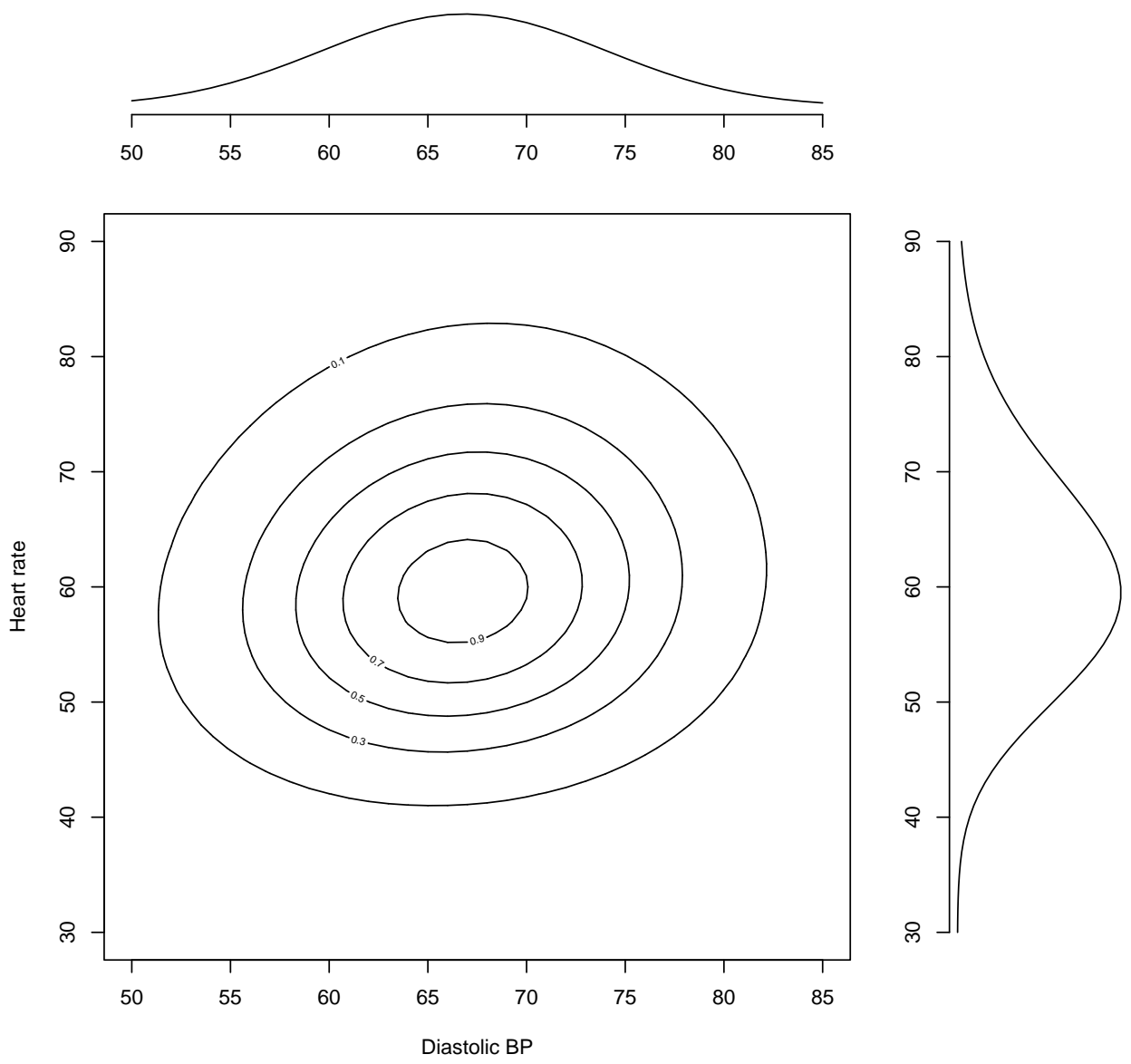

Figure 9: Fitted univariate and bivariate marginal distributions of the heart rate (in beats/min) and of the diastolic $\mathrm{BP}$ (in $\mathrm{mmHg}$ ) for a male with $35 \mathrm{ng} / \mathrm{mml}$ of drug in the plasma. 

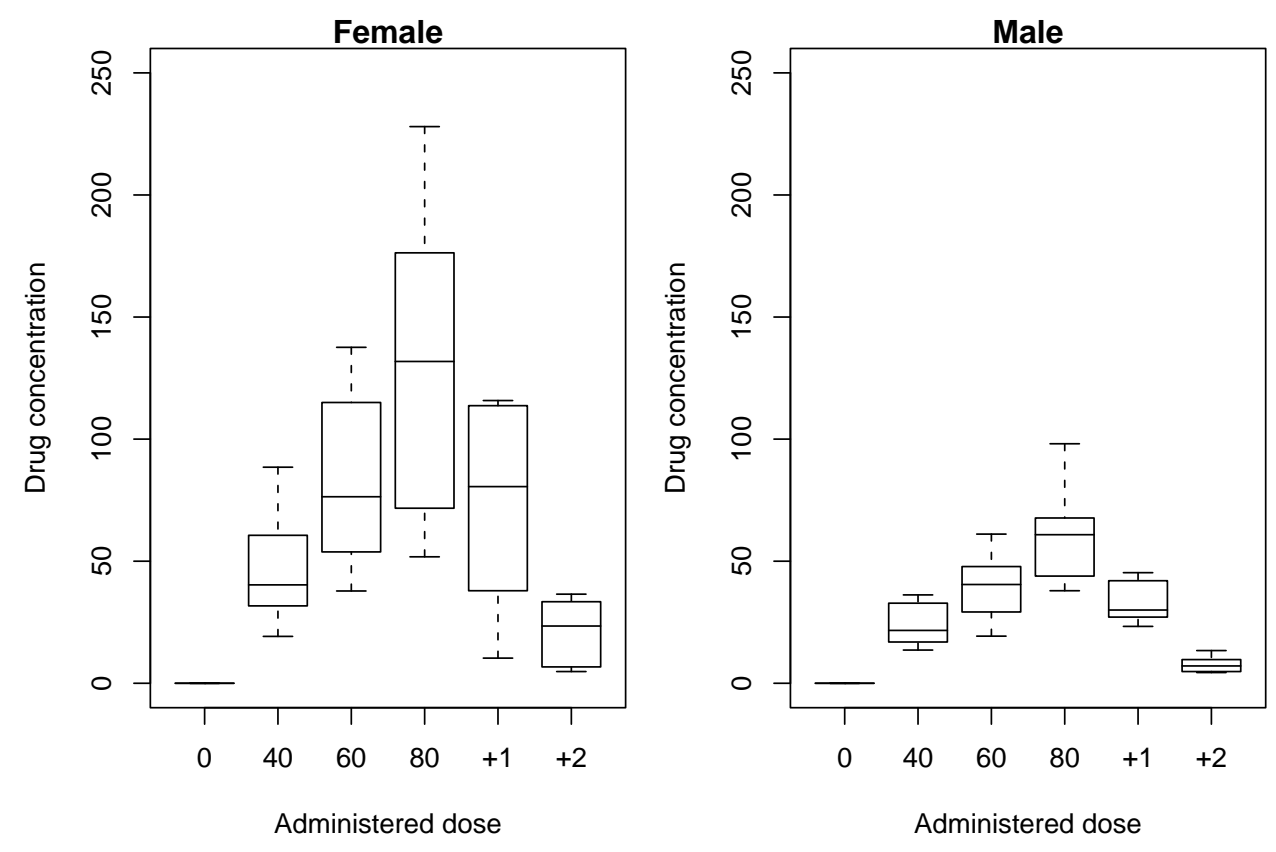

Figure 10: Steady state concentration of the drug in the plasma (in $\mathrm{ng} / \mathrm{ml}$ ) 4 hours after the indicated administered dose (in $\mathrm{mg}$ ) and then 1 and 2 days after stopping the drug administration. 


\begin{tabular}{c|c|c|cc|cc}
\multirow{2}{*}{ Response } & \multicolumn{2}{|c|}{ Diastolic BP } & \multicolumn{2}{c}{ Systolic BP } \\
Distribution & \multicolumn{2}{|c|}{ Normal } & \multicolumn{2}{c}{ Log-Student } & \multicolumn{2}{c}{ Heart rate } \\
\hline & MLE & s.e. $\Delta$ Dev & MLE $\quad$ s.e & $\Delta$ Dev & MLE & s.e.
\end{tabular}

Location parameters

\begin{tabular}{|c|c|c|c|c|c|c|c|c|c|}
\hline$\lambda_{0 M}$ & 65.4 & (1.11) & & 4.72 & $(0.03)$ & & 61.1 & (3.37) & \\
\hline$\Delta \lambda_{0 F}$ & 0 & & {$[-0.22]$} & 0 & & {$[-0.83]$} & 15.0 & $(4.62)$ & {$[+7.27]$} \\
\hline$\lambda_{1 M}$ & 0.041 & $(0.013)$ & {$[+7.18]$} & 0.00031 & $(0.00016)$ & {$[+3.42]$} & 0 & & {$[-0.22]$} \\
\hline$\Delta \lambda_{1 F}$ & 0 & & {$[-1.85]$} & 0 & & {$[-1.45]$} & 0 & & \\
\hline
\end{tabular}

\section{Dispersion parameters}

\begin{tabular}{c|ccc|ccc|ccc}
\hline$\delta_{0 M}$ & 1.30 & $(0.211)$ & & -2.43 & $(0.14)$ & & -3.65 & $(0.30)$ \\
$\Delta \delta_{0 F}$ & 0 & & {$[-0.01]$} & 0 & & {$[-0.03]$} & 0 & {$[-0.40]$} \\
$\Delta \delta_{C}$ & 0.61 & $(0.18)$ & {$[+7.73]$} & 0 & {$[-0.66]$} & 0 & {$[-0.22]$} \\
$\delta_{1 M}$ & 0.0017 & $(0.0011)$ & {$[+2.98]$} & 0 & {$[-0.03]$} & 0 & {$[-0.10]$} \\
$\Delta \delta_{1 F}$ & 0 & & {$[-0.02]$} & 0 & & -0.0049 & $(0.0019)$ & {$[+5.80]$}
\end{tabular}

Tail parameter

\begin{tabular}{l|ll|lll|l}
\hline$v$ & $+\infty$ & {$[-0.67]$} & 7.14 & $(3.51)$ & {$[+4.13]$} & -
\end{tabular}

Dependence parameters

\begin{tabular}{c|ccc|ccc|ccc}
\hline$\rho_{h}$ & 0.68 & $(0.06)$ & {$[+113.7]$} & 0.56 & $(0.14)$ & {$[+79.58]$} & 0.67 & $(0.10)$ & {$[+32.90]$} \\
$\rho_{s}$ & 0 & & {$[-0.67]$} & 0 & & {$[-0.76]$} & 0.30 & $(0.10)$ & {$[+7.85]$}
\end{tabular}

Likelihood

\begin{tabular}{|c|c|c|c|}
\hline$-2 \log (L)$ & 846.53 & 1039.01 & 937.48 \\
\hline \# Par. & 6 & 5 & 6 \\
\hline $\mathrm{AIC}$ & 858.53 & 1049.01 & 949.48 \\
\hline
\end{tabular}

Table 1: MLEs, standard errors (in brackets), deviance change (in square brackets: $-2 \log (L)$ increase when the corresponding parameter is fixed or $-2 \log (L)$ decrease when it is estimated) and likelihood for the selected marginal models for the three series of responses. 
Dependence parameters

\begin{tabular}{l|cccccc}
\hline & \multicolumn{2}{|c}{ HR-SBP } & \multicolumn{2}{c}{ HR-DBP } & \multicolumn{2}{c}{ SBP-DBP } \\
& MLE & se & MLE & se & MLE & se \\
\hline$\kappa_{0}^{M}$ & 0.090 & $(0.795)$ & 2.346 & $(0.642)$ & 0.273 & $(0.959)$ \\
$\Delta \kappa_{C}^{M}$ & 0.115 & $(0.827)$ & -1.905 & $(0.682)$ & 1.177 & $(0.981)$ \\
\hline$\kappa_{0}^{F}$ & -0.481 & $(0.805)$ & 1.415 & $(0.726)$ & 0.686 & $(0.768)$ \\
$\Delta \kappa_{C}^{F}$ & 0.121 & $(0.843)$ & -1.451 & $(0.768)$ & -0.003 & $(0.803)$ \\
& & & & & \\
\hline
\end{tabular}

Table 2: MLEs (standard errors) of between responses dependence parameters and likelihood for the full multivariate model. 
Dependence parameters

\begin{tabular}{c|ccc|ccc|ccc}
\hline & \multicolumn{2}{|c|}{ HR-SBP } & \multicolumn{3}{c|}{ HR-DBP } & \multicolumn{3}{c}{ SBP-DBP } \\
& MLE & se & $\Delta$ Dev & MLE & se & $\Delta$ Dev & MLE & se & $\Delta$ Dev \\
\hline$\kappa_{0}^{M}$ & 0 & - & {$[-0.12]$} & 1.894 & $(0.406)$ & {$[+9.81]$} & 0 & - & {$[-0.13]$} \\
$\Delta \kappa_{C}^{M}$ & 0 & - & {$[-0.21]$} & -1.671 & $(0.432)$ & {$[+7.80]$} & 1.447 & $(0.206)$ & {$[+33.35]$} \\
\hline$\kappa_{0}^{F}$ & 0 & - & {$[-0.61]$} & $=\hat{\kappa}_{0}^{M}$ & & {$[-0.94]$} & 0.717 & $(0.209)$ & {$[+10.10]$} \\
$\Delta \kappa_{C}^{F}$ & 0 & - & {$[-1.27]$} & $=\Delta \hat{\kappa}_{C}^{M}$ & & {$[-0.59]$} & 0 & - & {$[-0.01]$}
\end{tabular}

\section{Likelihood}

\begin{tabular}{lr}
\hline$-2 \log (L)$ & 2769.34 \\
\# Marginal parameters & 17 \\
\# Dependence parameters & 4 \\
AIC & 2811.34
\end{tabular}

Table 3: Between responses dependence parameters MLEs $(-2 \log (L)$ increase when the corresponding parameter is fixed (usually at 0 ) or $-2 \log (L)$ decrease when it is estimated) for the selected multivariate model.

Fitted Kendall's tau

\begin{tabular}{lc|ccc}
\hline Sex & Drug in plasma & HR-SBP & HR-DBP & SBP-DBP \\
\hline Male & No & 0 & 0.53 & 0 \\
& Yes & 0 & 0.07 & 0.42 \\
\hline \multirow{2}{*}{ Female } & No & 0 & 0.53 & 0.22 \\
& Yes & 0 & 0.07 & 0.22
\end{tabular}

Table 4: Fitted Kendall's tau for pairs of responses in the selected multivariate model. 